33 cases, 27 in girls and six in boys, and Brun's figures about correspond with these. In one case recorded by Lop the peritonitis followed delivery and pneumococci were found in the lochia. Some cases are, no doubt, manifestations of a general pneumococcus blood infection, whereas others, which start as a peritonitis, die from a subsequent pneumococcus septicæmia.

As regards the diagnosis, the chief points which have been noted to distinguish it from other forms are the diarrhoea, the great mental excitement, and the odourless character of the pus. But, as pointed out by Charrin and Veillon, the peritoneum may become infected secondarily by the bacillus coli communis and in such cases the characteristic odour of this bacillus may be noted, even in cases of primary pneumococcus peritonitis. The diagnosis eventually rests upon the examination of the exudate by the microscope, by cultures, and by animal inoculation.

Bibliography.- Weichselbaum, Centralblatt fiir Bakteriologie und Parasitenkunde, 1889 ; Boulay et Courtois Suffit, Bulletin de la Société Médicale res Hópitaux, 1890 ; Charrin et Veillon, Comptes Rendus des Séances de la Société de Biologie, 1893; Arnozan et Cassaet, Bulletin de la Société Médicale des Hôpitaux. 1895; Brault, Bulletin de la Société de Chirurgie, 1894; Gazette Hebdomadaire. 1898 : Bouilly,
Bulletin de la Société de Chirurgie, 1897; Brun, La Presse Médicale, 1897; Walther, ibid., 1897; Dieulafoy, Cliniques de l'Hôtel Dieu, vol. i., 1898; Ḿnetrier et Legroux, Bulletin de la Société Médicale des Hôpitanx, 1900; Lop, Gazette des Hôpitaux, 1900; Michaut, ibid., 1901 ; Bryant, Brit. Med. Jour., 1901 ; Quervain, Correspondenzblatt fiir Schweizer Aerzte, 1902 ; Vanderlinden, Centralblatt fur Chirurgie, 1902: Lennander, Deutsche Zeitschrift für Obirurgie, 1902 ; Jensen, Archiv fir Klinische Chiruroie, 1903; Brunn, Deutsche Gesellschaft fiir Ohirurgie. 1903; Waldo, Brit. Med. Jour., 1904; Duckworth and Marsh, Transactions of the Clinical Society of London, 1904; Shaw and French, Brit. Med. Jour., 1904; Boyd, Brit. MeA. Jour, 1904 Hawkins, The LANCFT March 4th, 1905, p. 568; Staple, ThE LasceT, June 10th, 1905, p. 1577:

Devonshire-street, $W$

\section{NECROSIS OF THE ENTIRE RENAL CORTEX OF BOTH KIDNEYS}

BY H. GAIRNS LLOYD, M.D. EDIN., F.R.O.S. FDIN., FONORARY SURGEON TO THE MIDWIFERY DEPARTMENT, WOMEN'S HOSPITAL, MELBOURNE; LATE ASSISTANT MASTER, ROTUNDA
HOSPITAL, DUBLIN.

AT a meeting of the Pathological Society of London on Oct. $17 \mathrm{th}, 1905,1$ Dr. W. S. A. Griffith and Dr. W. P. Herringham reported a case under the above heading. The specimens were obtained from a multipara after confinement and the authors assert that only one similar case can be found in literature. I therefore ask space to record the following which differs in certain particulars and has several points of interest.

A woman, aged 39 years, a multipara, was admitted under my care at the Women's Hospital, Melbourne, some three weeks short of her full time with a history, confirmed by examination, of accidental hæmorrhage. Her previous con finement was instrumental; otherwise there was nothing worthy of note. She gave a history of headaches and impaired vision, with a diminished secretion of urine for the past two weeks. Labour had commenced and the membranes were ruptured. With good pains a female stillborn fotus weighing six and a half pounds was expelled three hours after admission and was followed at once by the placenta and retro-placental clot weighing one and a half pounds. Two hours after delivery she had an eclamptic convulsion and during the next five hours three others but no more followed. The skin responded very well to vapour baths but the bowels were moved with difficulty, cathartics and enemata at first having no effect, though later there were frequent fluid stools. During the first 24 hours from admission only six and a half drachms of urine were passed. This contained a quantity of albumin and one grain to the ounce urea. The microscope showed no casts but some blood, chiefly white corpuscles. For the next 24 hours only 40 minims of urine were obtained and from this on to the ninth day not one drop was secreted and then four drachms were drawn off. The blood pressure in the radial artery was equivalent to 188 millimetres of mercury, on the second day 156 , and the lowest reading throughout was 120 millimetres. Local applications were used without avail, the usual diuretics, and diuretin and erythrol tetranitrate, and $I$ believe that the patient was kept alive so long as 11 days by means of repeated vapour baths to which the skin always responded well. On the seventh day there was some vomiting and on the eighth day intermittent uræmic twitchings commenced. The twitchings continued at intervals, though at no time violently, till the time of death which occurred on the rnorning of the eleventh day, consciousness continuing to the end. The temperature was normal or subnormal throughout.

Necropsy. - At the post-mortem examination the liver was found to be large, tough, and fibrous, with capillary streakings under the capsule. The spleen was large and tough, of chronic interstitial type. There was some hypertrophy of the left ventricle of the heart. The uterus was large, subinvoluted, with a large cavity containing some blood-coloured semi-fluid material with highly ammoniacal odour. In addition to the very uncommon condition of the kidneys, and no doubt in consequence of them, two points of great interest are that after a considerable ante-partum hæmorrhage and delivery eclamptic convulsions should occur and that the subject of these convulsions should have previously borne six children without such trouble arising, and the last of these at a very recent date. We altogether failed to obtain a history of any marked falling off in the amount of urine passed previously to admission.

Pathological Report on Kidneys and Liver, by Constanch FuLIS, M.D., Demonstrator in Pathology, University of Melbourne. - The organs have been immersed in formalin and some alteration has taken place. With regard to the kidneys, both are larger than normal, but without any alteration in shape. The right kidney weighs eight ounces and the left nine and a half ounces. The capsule seems to have been adherent to surrounding tissues but not to the kidney. The surface of the organ is smooth, of buff colour, mottled with numerous pin-head hæmorrhages. At parts the capsule was adherent and the tissues more decidedly yellow, tending to soften after a necrotic process. On section the cortex appears to retain its normal width; almost the whole of the cortical area presents the yellow bloodless appearance and firm consistence typical of coagulation necrosis. This change begins very close to the surface, only a fine line of purplish cortex still persisting immediately beneath the capsule. Even this line is absent at parts. The necrosis extends widely through the cortex, the depth varying from a quarter to half an inch. Its deep boundary is everywhere sinuous and along the edge of the necrotic area there is a line of tissue of a deeper red colour which microscopically is seen to be due to engorgement and hæmorrhage. Frequently the necrosis extends into the columns of Bertini. The large arteries do not show any marked thickening of their coats, nor is there any decided atheroma, though the intima is slightly ridged. No thrombi are found in any of the larger arteries. There is no dilatation of either pelvis or ureter. This description applies equally to both kidneys. With regard to the liver, only a small portion of this organ was sent to me. It shows a smooth capsule. On section the surface is pale and the centres of the lobules are slightly congested.

Mioroscopic examination.--Sections of the kidney stained with methylene blue and eosin failed to demonstrate the presence of any micro-organisms. In the necrotic area the tubular epithelium is destroyed and appears as a granular mass staining faintly with eosin. All division into individual cells is lost and no nuclei are visible. The same description applies to the Malpighian tufts in this area. The spaces between the tufts and tubes are infiltrated with numerous cells of leucocytic type. These are all undergoing fragmentation and no definite nuclear shapes are present though the fragments stain deeply with hæmatoxylin. At the boundary of the necrosed area numercus hæmorrhages have occurred. In arteries corresponding in size and position to the inter lobular vessels red thrombi are almost universal. The thrombi stain with eosin and present an appearance as of a mass of agglutinated red corpuscles. There is no layer of leucocytes next the vessel wall but a few nuclei are scattered through the thrombi. In the portion of cortex unaffected by necrosis the epithelium of the tubules stains feebly and shows evidence of cloudy swelling. Hyaline and epithelial casts are of frequent occurrence. The tufts contain an abnormal number of nuclei, they are often lobulated, and Bowman's capsule is thickened. 'The vessels of the boundary zone showed no marked change in their coats but the tiny arterioles have thickened walls and relatively small lumina. The increase of interstitial tissue is 
very marked; it contains numerous oval and elongate nuclei and fine fibres which now form a very open meshwork possibly due to adematous distension. Leucocytes of varying type are present, both singly and in patches. The variable layer of non-necrosed tissue just beneath the capsule presents similar features, including engorgement anrl hæmorrhages. In the pyramidal tissue the epithelium of the straight tubules is less affected and still stains selectively. Casts are frequent. There is considerable engorgement and here also the interlobular connective tissue is increased in amount.

As to the liver the nuclei stain with hrematoxylin, showing several large chromatin granules. The protoplasm is lumpy and granular, presenting the appearance of cloudy swelling. Staining with sudan iii. fails to demonstrate more than an occasional globule of fat, nor can free iron be found after treatment with dilute hydrochloric acid and potassium ferrocyanide. The capillary spaces within the lobules are dilated but are not engorged, containing an occasional red and white corpuscle. The intralobular veins are dilated but empty. In the portal canals there is slight increase in the connective tissue but within the lobules little inter-cellular fibrosis. The small branches of the hepatic artery have slightly thickened walls. No hæmorrhages and no marked pathological changes were thus observed in the liver.

Remarks.-The kidneys are macroscopically identical with those described by Rose Bradford in the Journal of Pathology and Bacteriology for 1898, although he does not mention the small $\mathrm{k}$ morrhages found in this case. Microscopically they differ in that in his case there was a widespread endarteritis withont interstitial fibrosis, while here the opposite obtains. The thrombi in his description are also of different character. In this case the thrombi seem to me to indicate the possibility of some agglutinin, though why it should operate only in arteries in such a limited area is mysterious. The size and weight of the kidneys and the large amount of interstitial fibrosis point to some previous renal affection of the large pale type. The condition of the liver would appear to be due to a secondary toxic effect. The fibrosis might be due to syphilis, either congenital or acquired, but the histological evidence for or against is quite inconclusive.

Melbourne.

\section{A CASE OF ACUTE HæMORRHAGIC PANCREATITIS.}

BY G. RUTHERFORD JEFFREY, M.B., Cr.B. GlasG., SENIOR ASSISTANT PHYSICIAN, AYR DISTRICT ASYLUN.

THE following case seems to be sufficiently rare to be worthy of record. The patient, a married woman, aged 60 years, was admitted into the Ayr District Asylum on Nov. 3rd, 1898, suffering from chronic mania, although at the commencement of her illness 26 years ago her mental condition was one of melancholia. She had always enjojed good bodily health, was the mother of five children, and was a stoutly built, robust, and well-nourished woman. There was no history of indigestion, colic, or jaundice and her family history was unimportant. Until Friday, Sept. 22nd, 1905 , she was perfectly well physically and it was only on the evening of that day that her illness commenced. She was in her usual state of health on the Friday and had taken her food well, but in the evening she complained of feeling a little sick, on account of which she was sent to bed. About one and a half hours afterwards the feeling of nausea developed into actual vomiting, accompanied by great abdominal pain, and when seen at 10 P.M. her condition was as follows. She was in a state of great collapse and was evidently in great suffering. She was retching and vomiting profusely, the vomited matter soon becoming bilious in character. She complained of great abdominal pain but was not able to locate it to any special area of the abdomen. On one occasion she described the pain as somewhat " bearing in character and as if something was moving." Her temperature was $98^{\circ} \mathrm{F}$. ; her pulse, which was weak, rapid, and fluttering, numbered 120 beats to the minute. Her tongue was dry and furred and her breath was very foul. On palpation the abdomen was universally "tender" and percussion revealed some distension of the stomach and tranver: colon.
The romiting and retching continued at short intervals during the night, but the pain was somewhat alleviated by the application of hot fomentations to the abiomen. The patient's condition on the following morning was much the same except that she was weaker and was as well slightly jaundiced. The vomiting and retching-which resisted all treatment-continued throughout the day, whilst the pain in the abdomen remained much about the sime. In the evening she was more collapsed, her pulke was 130, and her temperature, which in the morning was $98^{\circ}$, had now risen to $100^{\circ}$. Her tongue was dry and coated with a thick, dirty fur, her extremities were cold and her brow was covered with a cold "clammy" swe 1 t. The distension of the stomach and colon was more marked; the liver was now distinctly palpable and extended well be!ow the costal margin. Throughout the Sunday the patient's condition presented little change. The pain became worse, necesstating the use of morphine, the retching and vomiting continued, she complained of great thirst, and she had now sorne diarrhoa. There was no marked change in the temperature but her pulse was almost imperceptible. She became gradually weaker, the pain and vomiting continued, and she died at 2.20 P.M. on Monday, in less than three days from the commencement of her illness.

Necropsy.-On Sept. 26th a post-mortem examination was made. The body was that of a well-nourished woman. Postmortem lividity and rigidity were present. The thorax and abdomen were opened in the usual way ard the viscera were exposed. The organs, in situ, showed marked distension of the stomach and transver:e colon, the liver was sumewhat enlarged, and there was marked fatty infiltration of the omentum. The heart was healthy, as also were the lungs, with the exception of a few old pleural adhesions at the right apex and slight congestion of the right lower lobe. On passing the hand into the abdomen a lirge firm mass was detected extending transver ely across the abdomen, firmly adherent to all the surrounding structures, and in position occupying that of the pancreas. With difficulty the mass was separated from the surrounding structures and it weighed 11 ounces. The surface was dark-red in colour-in some places almost black-and on section only in parts could the true pancreatic tissue be recognised, the whole substance being much congested and in places showing areas of necrosis. The omentum and mesentery were abnormally fatty and on both were seen numerous white specks (the "fat necrosis" of Baker). The liver, which was adherent to the under surface of the diaphragm, weighed 51 ounces, and was enlarged, pale, soft, and fatty. The gall-bladder, the walls of which were thickened, contained a small quantity of bile, and as well four mulberry calculj, varying in size from that of a cherry to that of a pea. The spleen was congested and weighed six and a half ounces. 'Tbe right kioney weighed ten ounces, the left seven ounces, and both organs showed well-marked cloudy swelling. With the exception of the distension of the stomach axd transverse colon the gastrointestinal tract was normal.

Microscopieal examination.-Owing to changes-necrotic or post-mortem-the sfetions prepared did not stain properly and the glandular tissue itself stained but faintly and ciffusely. In various parts of the gland eviclence was found of multiple, more or less recent, small bæmorrhages from the capillar:es, all the vessels were engorged, and many of the larger vessels, both arteries and veins, showed signs of an acute inflammatory process. There was no general invasion of the tissues by micro-organisms but the larger pancreatic ducts were loaded with a bacillus probably the bacillus coli communis.

Comments -- As regards the etiology of the disease not much seems to be known. Osler in his "Text-kook of Medicine," : ays: "Many of the patients are addicted to alcohol, others had suffered occasionally with severe pains and vomiting or with gall-stone colic." I The pathology of the disease is obscure. Some writers emphasise the association of this condition with gall-stones and certainly in the case above quoted stones were found in the gall-kladder. In Osler's "Textbork of Medicine" a case is rtlated in which "a small calculus had lodged in the diverticulum of Vater, c'osing its duodenal orifice and converting the common bile-duct and the duct of Wirsung into a closed channel ; bile, finding its way into the pancreas, had caused bæmorrhagic inflammation." Other writers again assert that bacterial infection has something to do with the immediate carse. In the case 